\title{
Erratum: Exciton-polaron spectral structures in two-dimensional hybrid lead-halide perovskites [Phys. Rev. Materials 2, 064605 (2018)]
}

Stefanie Neutzner, Félix Thouin, Daniele Cortecchia, Annamaria Petrozza,

Carlos Silva ${ }^{\circledR}$, and Ajay Ram Srimath Kandada

(2) (Received 19 April 2020; published 20 May 2020)

DOI: 10.1103/PhysRevMaterials.4.059901

\section{APPENDIX A: MODIFIED ELLIOTT FORMULA}

We report a typographical error in Eq. (A1). The correct expression is the following:

$$
\begin{aligned}
\alpha(\hbar \omega)= & \alpha_{\mathrm{exc}}+\alpha_{\mathrm{cont}} \\
= & \alpha_{0}\left(\sum _ { n = 1 } ^ { \infty } \left\{\sum_{j=2}^{j=4} \frac{X_{j} E_{j}}{\left(n-\frac{1}{2}\right)^{3}} \operatorname{sech}\left(\frac{\hbar \omega-E g+\frac{E_{j}}{\left(n-\frac{1}{2}\right)^{2}}}{\Gamma_{\mathrm{exc}}}\right)\right.\right. \\
& \left.+\sum_{m_{i}} \frac{X_{1} E_{0}}{\left(n-\frac{1}{2}\right)^{3}} \operatorname{sech}\left(\frac{\hbar \omega-E g+\frac{E_{0}}{\left(n-\frac{1}{2}\right)^{2}}}{\Gamma_{\mathrm{exc}}}\right) \delta\left[\hbar \omega-\left(E_{g}-\frac{E_{0}}{\left(n-\frac{1}{2}\right)^{2}}\right)-\sum_{i} m_{i} \hbar \omega_{i}\right] \prod_{i} \frac{e^{-S_{i}} S_{i}^{m_{i}}}{m_{i} !}\right\} \\
& \left.+\int_{E_{g}}^{\infty} \operatorname{sech}\left(\frac{\hbar \omega-\varepsilon}{\Gamma_{c}}\right) \frac{1}{1+e^{-2 \pi \sqrt{\frac{E_{0}}{\hbar \omega-E_{g}}}}} \frac{1}{\left[1-\frac{\alpha 8 m_{*}}{\hbar^{4}}\left(\varepsilon-E_{g}\right)\right]} d \varepsilon\right) .
\end{aligned}
$$

The analysis presented in the paper, stemming from this expression, was performed with its correct form, and the error in the published paper does not affect the conclusions in any manner. 